\title{
A Broken Drill-bit Fragment Causing Severe Radiating Pain after Cervical Total Disc Replacement: A Case Report
}

\author{
Chong-Suh Lee, Sung-Soo Chung, Jae-Chul Park, \\ Seong-Kee Shin, Yong-Serk Park, Kyung-Chung Kang \\ Department of Orthopaedic Surgery, Samsung Medical Center, \\ Sungkyunkwan University School of Medicine, Seoul, Korea
}

\begin{abstract}
This is a case report of a 38-year-old man with severe radiating pain on upper extremity after cervical total disc replacement (TDR). We faced an unusual complication that has not been reported yet. He underwent cervical TDR for left central disc protrusion on C5-6. After the surgery, preoperative symptom disappeared. However, at postoperative 1 year, he complained severe right-sided radiating pain that had a sudden onset. On postoperative X-ray, a metal fragment which seemed like a broken drill bit was shown within the spinal canal. To remove that, right-sided anterior microforaminotomy on C5-6 was performed and the metal fragment was removed successfully. After that, anterior fusion was done because the motion of the artificial disc was minimal and the removed structure seemed to attenuate stability during cervical motion. The operation resulted in prompt symptomatic relief. During cervical TDR, particular attention should be paid to the procedures that require using drill-bits.
\end{abstract}

Key Words: Drill-bit fragment, Complication, Cervical total disc replacement

\section{Introduction}

Total disc replacement (TDR) for spinal disorders is an attractive alternative to fusion surgery. Cervical TDR is popular due to the relatively easy technique in the procedure and the excellent postoperative results [1-3]. But it is possible that the increased number of cervical TDR procedures will result in a number of complications after the TDR operations.

Many reports have shown the complications after cervical TDR such as adjacent segmental diseases, heterotopic ossifications and decreases of segmental motion [4,5], yet there might be a few unusual complications, including aseptic loosening, delayed hyper-reactivity to metal ions, weakness of the bony endplate etc. [6,7].

In this report, we introduce an unexpected complication in the procedure using a TDR device, which had a keelbased design, and we show the entire course of our case.

\section{Case Report}

\section{Operation}

A 38-year-old man visited the clinic for a severe radiating pain on his left shoulder. He then underwent cervical TDR for a left central disc protrusion at the C5-6 level. We used a Prestige LP ${ }^{\circledR}$ cervical total disc device (Medtronic Sofamor Danek Inc., Memphis, TN, USA) according to a routine protocol [8]. After cervical TDR, the preoperative

Received Mar 9, 2010; 1st Revised Apr 26, 2010; Accepted May 19, 2010

Corresponding author: Kyung-Chung Kang, MD

Department of Orthopaedic Surgery, Samsung Medical Center, Sungkyunkwan University School of Medicine,

50 Irwon-dong, Gangnam-gu, Seoul 135-710, Korea

Tel : +82-2-3410-3509, Fax :+82-2-3410-0061, E-mail: futurespine@gmail.com 
radiating pain on his left arm and posterior neck pain had decreased. However, we noticed a unique metal signal on the immediate postoperative lateral X-ray of his cervical spine (Fig. 1). We discussed this unexpected abnormal finding, but we could not come to any conclusion. A re-operation for that abnormal metal signal was seriously considered and the patient was kept in the ward for close observation of his symptoms and neurological status, and he was permitted to have normal daily activity. At the postoperative 1 week, the patient was discharged after confirming no interval change on the sequential radiographic and clinical examinations.

\section{Postoperative course}

The patient came for follow-up at the 6-weeks, 3-months, and 6-months postoperatively. During these times, he noted there was no more radicular pain and only tolerable neck discomfort was present. We noted that there was a fine change in the position of the metal fragment. The patient was informed regarding the abnormal X-ray finding and we warned him that further migration that might give rise to serious symptoms.

A year after the surgery, he came to an emergency room showing a severe right-sided radiculopathy on his upper extremity. The pain was initially mild and it started after an uneventful outpatient visit. His radiating pain did not respond to any analgesic. A Philadelphia collar was prescribed and he was admitted to the general ward.

The patient described a sharp intolerable pain radiating from the posterior neck to the right elbow, which was aggravated by sitting or moving and it was relieved by lying without any movement. His neurological examination revealed normal findings, but at the extension of his neck, a severe radiating pain to his right upper limb occurred. Further examination was abandoned because of these problems.

\section{Re-operation}

A computed tomography scan was checked and a comparison with the previous image was made (Fig. 2). We found that the metal fragment had migrated posteriorly into the spinal canal and it could cause injury to neural structures. A decision to re-operate was made to remove the foreign material through an anterior cervical approach using an opposite right-side incision. A right uncinate process of the C6 vertebra was osteotomized with carefully protecting the vertebral artery. An anterior microforaminotomy was done with a high-speed burr on the right side of the implant at the C5-6 level. A $5 \mathrm{~mm}$ size opening was made and further

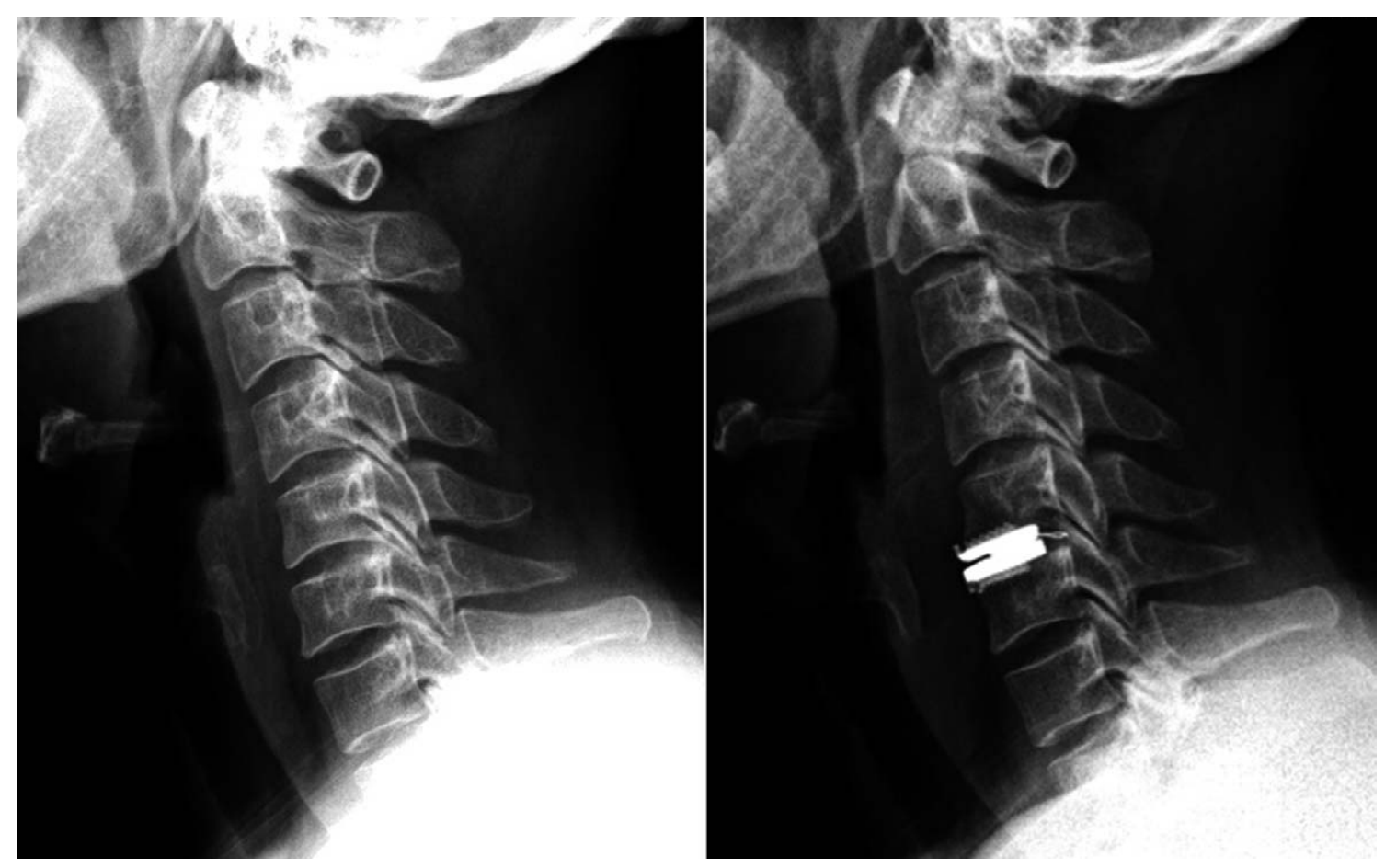

Fig. 1. On the immediate postoperative lateral X-ray, an unexpected metal signal was found in the posterior area of the implant. 


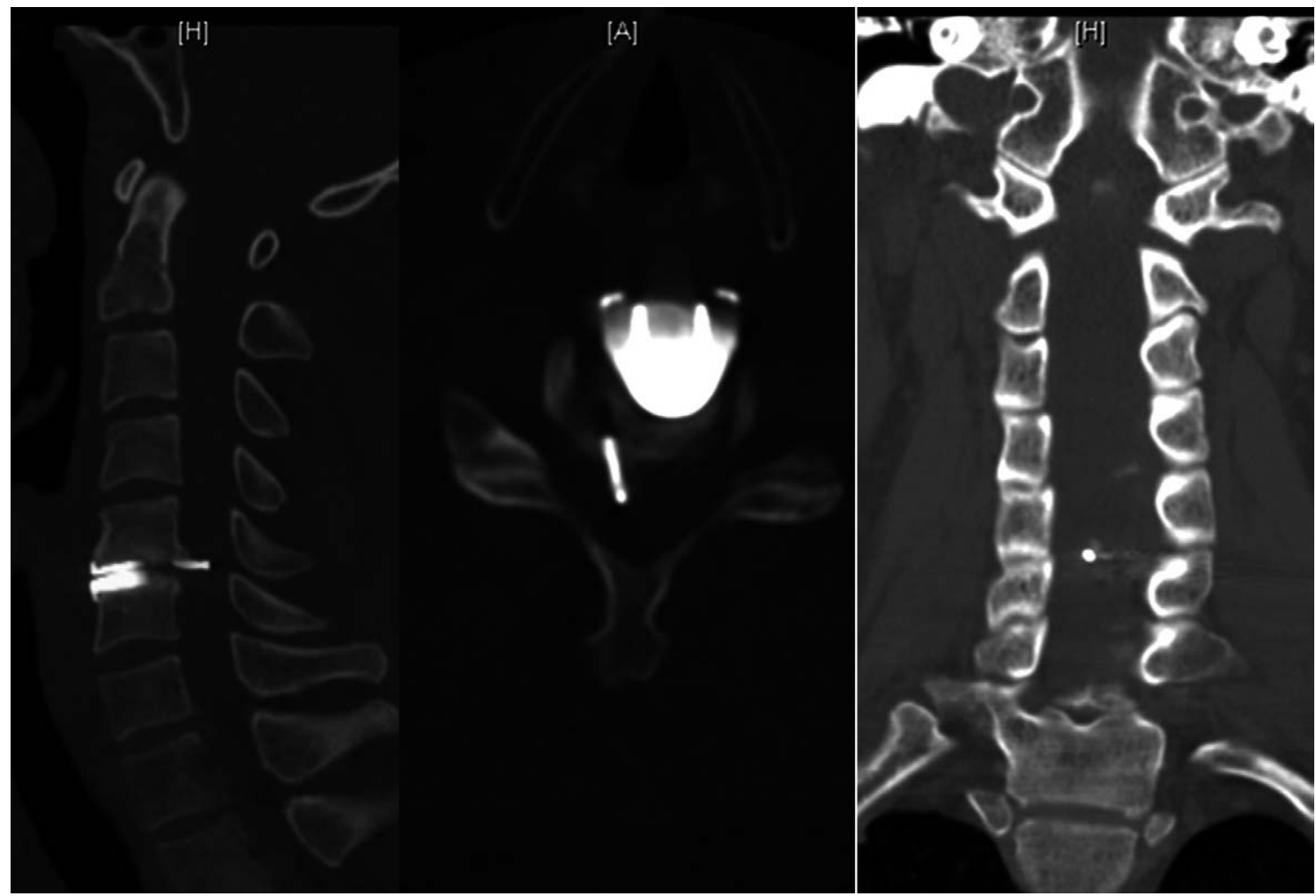

Fig. 2. On the postoperative $1 \mathrm{yr}$ computed tomography scan, the metal fragment was found in the right side of the spinal canal.

exploration was done under fluoroscopic guidance. When removing scar tissues around the implant, a cerebrospinal fluid (CSF) leak was observed and the metal fragment was expected to be in the close proximity. After deepening the dissection, a twinkling tip of the metal fragment was finally seen and removed with a small pituitary forceps (the socalled 'Black Pituitary Forceps'). After the removal of the metal fragment, C5-6 fusion was carried out with autologous iliac bone and an anterior cervical plate because the motion of the artificial disc was minimal when the remnant motion was carefully checked by distracting between the metal components in the C5-6 level, and the removed fragment seemed to attenuate the stability during cervical motion (Fig. 3). Removal of the arthroplasty implant during the fusion surgery was not considered because it would attenuate the dural deficit and unstable structure around the foramen. Gelfoam and fibrin glue were applied at the area of CSF leakage and a lumbar drain was inserted.

Postoperatively, the patient showed immediate symptomatic relief, but he suffered from a headache secondary to the dural tear and CSF leakage. Ambulation was permitted after 3 days of absolute bed rest. At the postoperative 7 days, all the symptoms were improved with administering only non-narcotic medication and he was discharged.

\section{Discussion}

Drilling procedures are necessary during TDR for a few cervical disc devices with a keel-based design [9]. The Prestige LP $^{\circledR}$ cervical disc prosthesis also needed predrilled channels in the endplates for the initial fixation that was made through two rails on either side of the midline. The predrilled channels were made with a 'rail cutter guide' with a diameter of only $1.5 \mathrm{~mm}$. Most of the drill-bits used in the cervical TDR were made by each individual TDR company and they are not standardized instruments, and these bits have a smaller diameter than the ordinary drillbits used in the usual procedures. Usually, surgeons are not concerned about the thickness of the drill-bits. This small diameter can make the drill-bit easily breakable during the TDR procedure. Therefore, during operations, the surgeons should carefully check the appropriate status of the channel before drilling and obtain an acceptable angle of the tip of the drill-bits. Repeated use of a drill-bit is another source of problems. Surgeons should keep in mind that these kinds of complications can happen and precaution must be taken to 


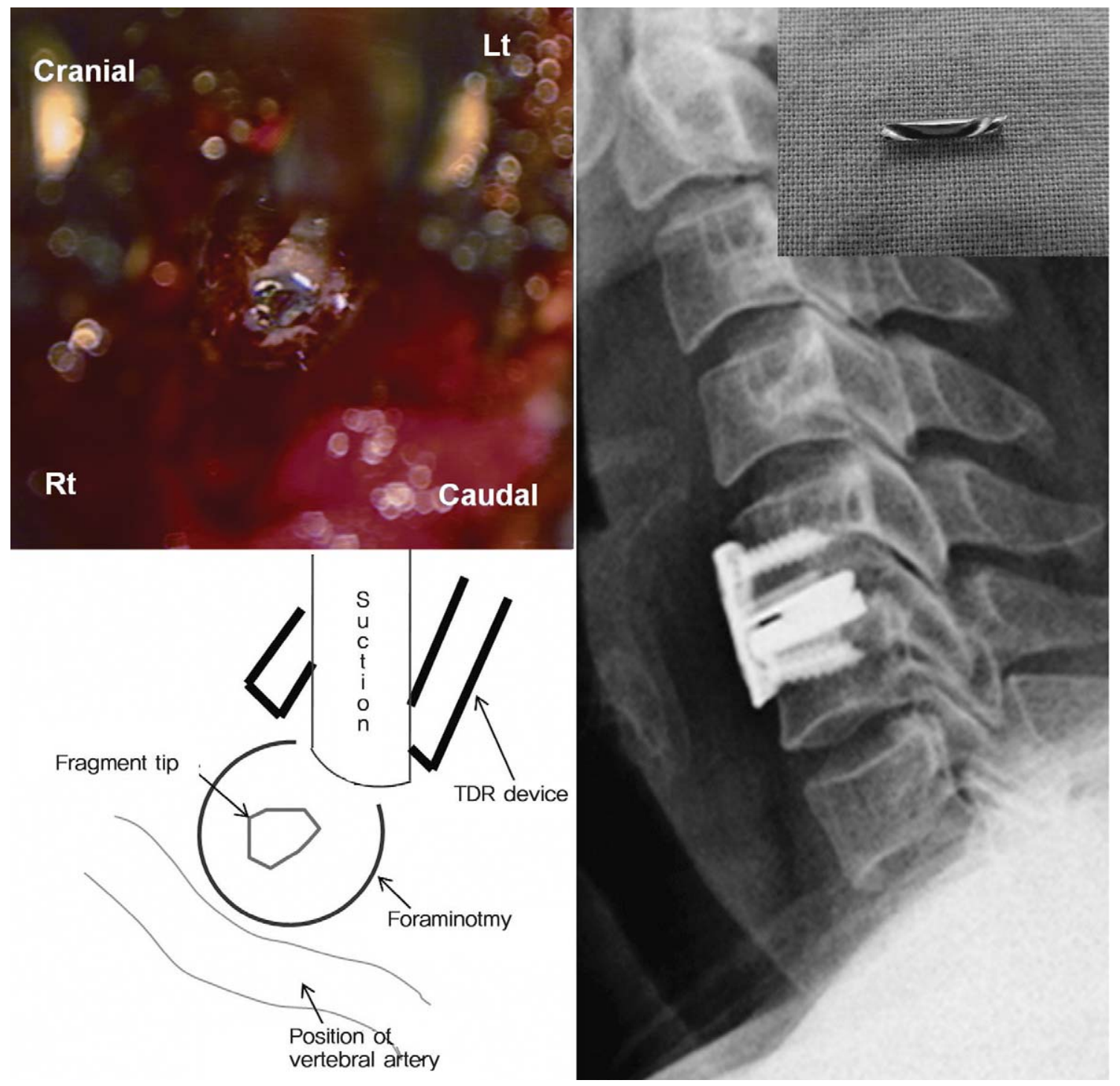

Fig. 3. The fragment tip was found and removed completely during the operation. Anterior fusion was done after that. The removed metal fragment is shown on the right photograph. Rt: Right, Lt: Left, TDR: Total disc replacement.

avoid a similar event during these procedures.

The authors and scrub personnel did not notice the metallic fragment during the operation. That was because of a surgeon's indifference except for the position and motion of the implant and the lower resolution power of the fluoroscopy that was used intraoperatively. We admit that it was a critical mistake not to notice that metal fragment during operation, but we want to inform others that these complications could occur again in other cases.

Another unsatisfied result was the decrease of the segmental motion. There are many factors that can cause decreased postoperative motion, but a slightly left-side position of the device and a small implant size for the disc space were considered the main factors preventing motion of the
TDR in this case.

Cases of cervical TDR are on the rise. Surgeons may be faced with more untoward events associated with the TDR procedure. Although there have been no previous reports about the problems with the drill-bits used during TDR, it was a serious problem in our case that could not be ignored. We wish to share this experience and recommend taking measures to avoid the occurrence of such serious problems.

\section{REFERENCES}

1. Sasso RC, Smucker JD, Hacker RJ, Heller JG. Artificial disc versus fusion: a prospective, randomized study with 2year follow-up on 99 patients. Spine (Phila Pa 1976) 
2007:32:2933-40.

2. Murrey D, Janssen M, Delamarter R, et al. Results of the prospective, randomized, controlled multicenter Food and Drug Administration investigational device exemption study of the ProDisc-C total disc replacement versus anterior discectomy and fusion for the treatment of 1-level symptomatic cervical disc disease. Spine J 2009;9:275-86.

3. Heller JG, Sasso RC, Papadopoulos SM, et al. Comparison of BRYAN cervical disc arthroplasty with anterior cervical decompression and fusion: clinical and radiographic results of a randomized, controlled, clinical trial. Spine (Phila Pa 1976) 2009;34:101-7.

4. McAfee PC, Cunningham BW, Devine J, Williams E, YuYahiro J. Classification of heterotopic ossification (HO) in artificial disk replacement. J Spinal Disord Tech 2003; $16: 384-9$.
5. Bartels RH, Donk R. Fusion around cervical disc prosthesis: case report. Neurosurgery 2005;57:E194.

6. Cavanaugh DA, Nunley PD, Kerr EJ 3rd, Werner DJ, Jawahar A. Delayed hyper-reactivity to metal ions after cervical disc arthroplasty: a case report and literature review. Spine (Phila Pa 1976) 2009;34:E262-5.

7. Zhang X, Ordway NR, Tan R, Rim BC, Fayyazi AH. Correlation of ProDisc-C failure strength with cervical bone mineral content and endplate strength. J Spinal Disord Tech 2008;21:400-5.

8. Mummaneni PV, Robinson JC, Haid RW Jr. Cervical arthroplasty with the PRESTIGE LP cervical disc. Neurosurgery 2007;60(4 Suppl 2):310-4.

9. Sekhon LH, Ball JR. Artificial cervical disc replacement: principles, types and techniques. Neurol India 2005; $53: 445-50$ 